\title{
Mediastinal Pleura
}

National Cancer Institute

\section{Source}

National Cancer Institute. Mediastinal Pleura. NCI Thesaurus. Code C94820.

The parietal pleura that lines the mediastinum. 